\title{
Staggered Chiral Perturbation Theory for Neutral $B$ Mixing
}

\author{
C. Bernard* \\ Department of Physics, Washington University, St. Louis, MO 63130, USA \\ E-mail: cb@wust 1. edu
}

\section{(The MILC Collaboration)}

I describe a calculation of $B$ meson mixing at one-loop in staggered chiral perturbation theory, for the complete set of Standard Model and beyond-the-Standard Model operators. The particular lattice representation of the continuum operators used by the Fermilab Lattice/MILC collaborations (and earlier by the HPQCD collaboration) turns out to be important, and results in the presence of "wrong-spin" operators, whose contributions however vanish in the continuum limit. The relation between staggered and naive fermions also plays a key role.

The $30^{\text {th }}$ International Symposium on Lattice Field Theory - Lattice 2012

June 24-29, 2012

Cairns, Australia

\footnotetext{
* Speaker.
} 


\section{Introduction}

The mixing of neutral $B$ mesons provides a fertile area for tests of the Standard Model and sensitivity to new physics. In order to take advantage of experimental measurements, lattice computations are as usual needed to determine the hadronic matrix elements of the effective weak operators. The mixing is dominated by the short distance contributions from the operators [1]

$$
\begin{array}{ll}
\mathscr{O}_{1} & =\left(\bar{b} \gamma^{v} L q\right)\left[\bar{b} \gamma^{v} L q\right], \quad \mathscr{O}_{2}=(\bar{b} L q)[\bar{b} L q], \\
\mathscr{O}_{4} & =(\bar{b} L q)[\bar{b} R q], \quad \mathscr{O}_{3}=(\bar{b} L q][\bar{b} q), \\
5 & =(\bar{b} L q][\bar{b} R q),
\end{array}
$$

where $\mathscr{O}_{1}$ through $\mathscr{O}_{3}$ control the mixing in the Standard Model, while $\mathscr{O}_{4}$ and $\mathscr{O}_{5}$ can appear in beyond-the-Standard Model (BSM) theories. Pairs of round or square parentheses in Eq. (1.1) indicate how the color indices are contracted, and $R$ and $L$ are the right and left projectors. Using Fierz transformations and parity, the mixing matrix element of any 4-quark operator with these quantum numbers can be written in terms of the matrix elements of these five "basis" operators; see, for example, Ref. [2] for details.

In a lattice computation, it is useful to be able to fit the lattice data to a version of chiral perturbation theory that includes the effects of the discretization errors associated with the lattice action. In two recent lattice calculations of $B$ mixing [3, 4], staggered light quarks are combined with nonstaggered heavy quarks using NRQCD [5] or the Fermilab action [6]. In such cases, the appropriate chiral theory is "rooted, heavy-meson staggered chiral perturbation theory" (rHMS $\chi$ PT) [7]. Here, I describe a calculation of $B$ mixing to one-loop order in rHMS $\chi$ PT. Roughly speaking, the calculation is to leading order in the heavy-quark expansion, although the largest $1 / m_{B}$ effects (the $B-B^{*}$ hyperfine splitting $\Delta^{*}$ and the $B_{s}-B_{d}$ flavor splitting) are also included. This is a systematic approximation in the power counting introduced by Boyd and Grinstein [8] and discussed recently in Ref. [9] for the lattice calculation of heavy-light meson decay constants.

The rHMS $\chi$ PT calculation needs to take into account the form of the lattice operator used to approximate the continuum one. References [3,4] construct the 4-quark operators as the local product of two local bilinears, each formed from a heavy antiquark field and a naive light-quark field. Both the use of naive fields and the local nature of the 4-quark operator influence the form of the corrections at one-loop.

\section{Detailed Structure of the Operators}

Heavy-light bilinears and 4-quark operators are made by converting a one-component staggered fermion $\chi(x)$ into a naive fermion $\Psi(x)$ following Ref. [10], and then coupling it locally to the heavy-quark field $Q(x)$ :

bilinear: $\quad \bar{Q}(x) \Gamma \Psi(x)$,

$$
\text { 4-quark operator: } \quad \bar{Q}(x) \Gamma \Psi(x) \bar{Q}(x) \Gamma^{\prime} \Psi(x) \text {, }
$$

where $\Gamma$ and $\Gamma^{\prime}$ are Dirac spin matrices. As we will see, bilinears of this form work exactly as desired in the lattice simulation, but the local product of the bilinears in the 4-quark operators introduces contributions from operators with wrong taste and spin (i.e., spin different from $\Gamma \otimes \Gamma^{\prime}$ ). 
The naive light-quark action can be written as four copies of the staggered action:

$$
\Psi(x)=\Omega(x) \underline{\chi}, \quad \Omega(x)=\gamma_{0}^{x_{0}} \gamma_{1}^{x_{1}} \gamma_{2}^{x_{2}} \gamma_{3}^{x_{3}} .
$$

Here $\underline{\chi}$ is a "copied" staggered field, with each Dirac component $\underline{\chi}_{i}$ separately having the staggered action. I will call the $S U(4)$ symmetry that acts on index $i$ "copy symmetry." Copy symmetry is an exact lattice symmetry, so copied and uncopied propagators are related by

$$
\left\langle\underline{\chi}_{i}(x) \underline{\bar{\chi}}_{i^{\prime}}(y)\right\rangle=\delta_{i, i^{\prime}}\langle\chi(x) \bar{\chi}(y)\rangle .
$$

where $\chi$ is the normal (uncopied) staggered field. An interpolating field $\mathscr{H}(x)$ for a heavy-light pseudoscalar meson is

$$
\mathscr{H}(x)=\bar{Q}(x) \gamma_{5} \Psi(x)=\bar{Q}(x) \gamma_{5} \Omega(x) \underline{\chi}(x) .
$$

In the simulations, $\mathscr{H}(x)$ is always summed over a time-slice (either explicitly, or implicitly by using translation invariance). To leading order in $a, Q(x)$ varies smoothly between neighboring spatial sites (up to gauge transformation), but $\underline{\chi}$ does not, due to taste doubling. Staggered fields are, however, smooth in the spin-taste basis on the doubled lattice, so we need to sum the field within a hypercube to expose the structure of the operators.

I focus first on the average of $\mathscr{H}(x)$ over a spatial cube. Let $x=(t, \boldsymbol{x})$ with $\boldsymbol{x}=2 \boldsymbol{y}$ even, and let $\eta=\left(\eta_{0}, \boldsymbol{\eta}\right)$ be a 4-vector with all components 0 or 1 . For $t$ even $(t=2 \tau)$,

$$
\begin{gathered}
\mathscr{H}^{(\mathrm{av})}(t, \boldsymbol{x})=\frac{1}{8} \sum_{\boldsymbol{\eta}} \bar{Q}(t, \boldsymbol{x}+\boldsymbol{\eta}) \gamma_{5} \Omega(2 \tau, \boldsymbol{\eta}) \underline{\chi}(2 \tau, 2 \boldsymbol{y}+\boldsymbol{\eta}) \cong \frac{1}{8} \bar{Q}(t, \boldsymbol{x}) \gamma_{5} \sum_{\boldsymbol{\eta}} \Omega(\boldsymbol{\eta}) \underline{\chi}(2 \tau, 2 \boldsymbol{y}+\boldsymbol{\eta}) \\
\cong \frac{1}{16} \bar{Q}(t, \boldsymbol{x}) \gamma_{5} \sum_{\eta}\left[\Omega(\eta) \underline{\chi}\left(2 \tau+\eta_{0}, 2 \boldsymbol{y}+\boldsymbol{\eta}\right)+(-1)^{\eta_{0}} \Omega(\eta) \underline{\chi}\left(2 \tau+\eta_{0}, 2 \boldsymbol{y}+\boldsymbol{\eta}\right)\right] .
\end{gathered}
$$

Inserted gauge links for point-split quantities are implicit. For $t$ odd, the result in Eq. (2.6) is the same except the last term changes sign. This is the usual oscillating state with opposite parity.

For simplicity, I assume from now on that the oscillating state is removed by the fitting procedure, and that all components of $x$ are even. Then

$$
\mathscr{H}^{(\mathrm{av})}(x) \rightarrow \frac{1}{16} \bar{Q}(x) \gamma_{5} \sum_{\eta} \Omega(\eta) \underline{\chi}(2 y+\eta) .
$$

To convert to a spin-taste basis, one can use [11]

$$
q_{i}^{\alpha a}(y)=\frac{1}{8} \sum_{\eta} \Omega^{\alpha a}(\eta) \underline{\chi}_{i}(2 y+\eta),
$$

where $\alpha$ is a spin index, $a$ is a taste index, and $i$ is a (trivially inserted) copy index. This implies that taste and copy indices are coupled in $\mathscr{H}$. We get (with spin indices implicit from now on)

$$
\mathscr{H}^{(\mathrm{av})}(x) \rightarrow \frac{1}{2} \bar{Q}(x) \gamma_{5} q_{i}^{a}(y) \delta_{i}^{a},
$$

where the arrow signifies that oscillating states and $\mathscr{O}(a)$ corrections are being dropped. Using Eq. (2.4), we see that the contraction of $\mathscr{H}$ with $\mathscr{H}^{\dagger}$ is automatically averaged over tastes:

$$
\left\langle\mathscr{H}(x) \mathscr{H}^{\dagger}\left(x^{\prime}\right)\right\rangle \sim \frac{1}{4}\left\langle\bar{Q}(x) \gamma_{5} q^{a}(y) \bar{q}^{a}\left(y^{\prime}\right) \gamma_{5} Q\left(x^{\prime}\right)\right\rangle .
$$


Note that the interpolating field constructed from the naive light quark gives us the desired pseudoscalar spin in the spin-taste basis (aside from the dropped oscillating state).

I now turn to the 4-quark operators constructed as in Eq. (2.2). The two bilinears are not separately summed over space, but they can be disentangled using

$$
\frac{1}{256} \sum_{K} \operatorname{tr}\left(\Omega(\eta) K \Omega^{\dagger}(\eta) K\right) \operatorname{tr}\left(\Omega\left(\eta^{\prime}\right) K \Omega^{\dagger}\left(\eta^{\prime}\right) K\right)=\delta_{\eta, \eta^{\prime}}
$$

where $K$ runs over the 16 independent Hermitian gamma matrices. Following the same kinds of manipulations as in Eqs. (2.5) through (2.9), one then finds

$$
\mathscr{O}_{n}^{(a v)}(x) \rightarrow \frac{1}{4} \sum_{K}\left(\bar{Q} \Gamma_{n} K q_{k}^{c} \bar{Q} \Gamma_{n}^{\prime} K q_{\ell}^{d}\right) K_{c k} K_{d \ell},
$$

where $\Gamma_{n}$ and $\Gamma_{n}^{\prime}$ are the Dirac spin matrices of the operators. Here the contributions with $K \neq I$ clearly have the wrong (undesired) spin, as well as non-trivial coupling of taste $(c, d)$ and copy $(k, \ell)$ indices. Putting Eq. (2.9) together with Eq. (2.12) and using copy symmetry gives

$$
\left\langle\mathscr{H}^{\dagger} \mathscr{O}_{n}^{(a v)} \mathscr{H}^{\dagger}\right\rangle \propto\left\langle D^{a c} D^{e d}\right\rangle K_{c a} K_{d e}+\text { (second equivalent contraction) }
$$

where $D^{a c}$ is the light-quark propagator (in a given background) for taste $a$ into taste $c$. When taste symmetry is exact, $\left\langle D^{a c} D^{e d}\right\rangle \propto \delta_{a c} \delta_{e d}$, so $K=I$, and only the correct spins contribute. This shows that 4-quark operators constructed as in Eq. (2.2) will have the correct (desired) spin in the continuum limit, as well as at tree level in rHMS $\chi \mathrm{PT}$, which respects taste symmetry. But, at one loop (and $a \neq 0$ ), taste-violations imply that $\left\langle D^{a c} D^{e d}\right\rangle$ is not necessarily proportional to $\delta_{a c} \delta_{e d}$. Thus one-loop diagrams will have contributions from wrong-spin operators.

\section{Calculation of the $\chi$ PT Diagrams at One Loop}

We now are ready to perform the calculation of the one-loop chiral corrections to matrix elements of the 4-quark operators. Aside from the wave-function renormalization diagram, which does not involve the operators and can be taken unchanged from the literature [7, 12], there are three diagrams, shown in Fig. 1.

Because copy and taste indices are coupled in Eq. (2.12), and because copy indices follow the quark contractions (Eq. (2.4)), we need to consider the quark flow of the meson diagrams in order to compute them. As an example, Fig. 2 shows a possible quark flow for each of the tadpole meson diagrams. These particular quark flows have "connected" pion propagators without hairpin vertices; other tadpole flows with either taste-singlet (physical) or taste-violating hairpin contributions are also possible.

Labeling the taste of the pion in the loop by $\Xi(\Xi=1, \ldots, 16)$, diagram Fig. 2(a) is proportional to $\Xi_{a f} \Xi_{f c} \delta_{e d} K_{c a} K_{d e}=[\operatorname{tr}(\mathrm{K})]^{2}$, where the factors of $K$ come from Eq. (2.12). In this case, only the correct spin contributes $(K=I)$. On the other hand, diagram Fig. 2(b) is proportional to $\Xi_{a d} \Xi_{e c} K_{c a} K_{d e}=\operatorname{tr}(\Xi \mathrm{K} \Xi \mathrm{K})$. If the propagator were independent of $\Xi$, the sum on tastes would again give $K=I$. Due to taste violations, however, the pion propagator depends on whether $\Xi$ is $\mathrm{P}$, A, T, V, or I. This implies that various $K$ values are possible, giving wrong-spin contributions. 


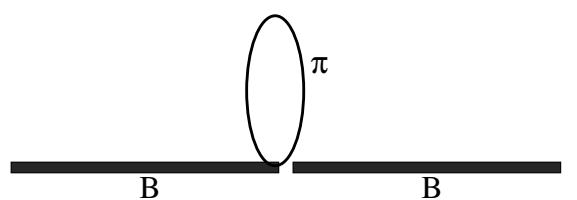

(a)

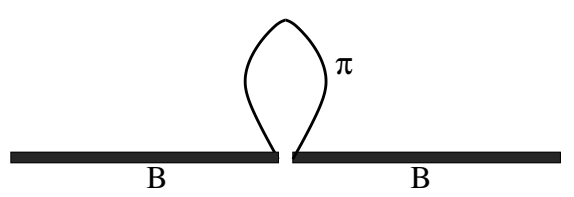

(b)

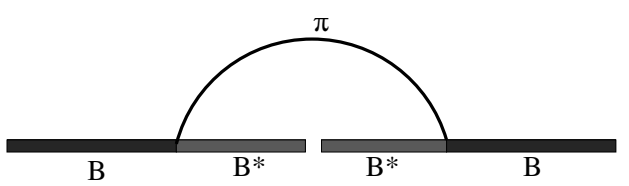

(c)

Figure 1: Meson-level chiral diagrams for the mixing matrix elements. The gap between the two $B$ (or two $B^{*}$ ) fields represents the insertion of the 4-quark operator. Diagrams (a) and (b) are tadpole diagrams, which are distinguished by whether the contracting pions come from the same meson field (loosely speaking, the same bilinear) or from different meson fields. Diagram (c) is the sunset diagram.

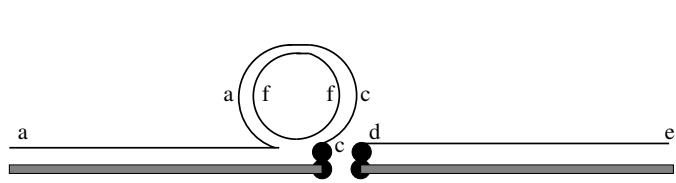

(a)

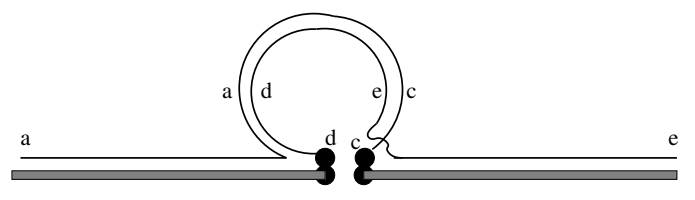

(b)

Figure 2: Examples of quark-flow tadpole diagrams. The filled, black circles represent the heavy and light quarks in the 4-quark operator. Diagram (a) contributes to the meson diagram Fig. 1(a), and diagram (b) contributes to diagram Fig. 1(b). Indices a-f label tastes.

The fact that wrong spins enter means that other operators appear, and they in turn have different chiral representatives than the original operator does. Fortunately, the basis of $\mathscr{O}_{1}, \ldots, \mathscr{O}_{5}$ is complete, and the chiral representatives of all these operators are given by Detmold and Lin [13]. Generalizing to operators with light-quark tastes $c, d$, we have

$$
\begin{aligned}
O_{1}^{x c ; x d} & =\beta_{1}\left[\left(\sigma P^{(b) \dagger}\right)_{x, c}\left(\sigma P^{(\bar{b})}\right)_{x, d}+\left(\sigma P_{\mu}^{*(b) \dagger}\right)_{x, c}\left(\sigma P^{*(\bar{b}), \mu}\right)_{x, d}\right] \quad[\text { or } c \leftrightarrow d], \\
O_{2(3)}^{x c ; x d} & =\beta_{2(3)}\left(\sigma P^{(b) \dagger}\right)_{x, c}\left(\sigma P^{(\bar{b})}\right)_{x, d}+\beta_{2(3)}^{\prime}\left(\sigma P_{\mu}^{*(b) \dagger}\right)_{x, c}\left(\sigma P^{*(\bar{b}), \mu}\right)_{x, d} \quad[\mathbf{o r} c \leftrightarrow d], \\
O_{4(5)}^{x c ; x d} & =\frac{\beta_{4(5)}}{2}\left[\left(\sigma P^{(b) \dagger}\right)_{x, c}\left(\sigma^{\dagger} P^{(\bar{b})}\right)_{x, d}+\left(\sigma^{\dagger} P^{(b) \dagger}\right)_{x, c}\left(\sigma P^{(\bar{b})}\right)_{x, d}\right] \\
& +\frac{\beta_{4(5)}^{\prime}}{2}\left[\left(\sigma P_{\mu}^{*(b) \dagger}\right)_{x, c}\left(\sigma^{\dagger} P^{*(\bar{b}), \mu}\right)_{x, d}+\left(\sigma^{\dagger} P_{\mu}^{*(b) \dagger}\right)_{x, c}\left(\sigma P^{*(\bar{b}), \mu}\right)_{x, d}\right] \quad[\text { or } c \leftrightarrow d] .
\end{aligned}
$$

where $P$ and $P^{*}$ are heavy-light meson fields, $\sigma$ is the pion field, and $x$ is the light flavor. The effect of copy indices is to enforce the contraction of the external quark of taste $a$ with the quark in the operator of taste $c$, and similarly for $e$ and $d$.

We then write the matrix element for operator $\mathscr{O}_{n}$ as

$$
\left\langle\bar{B}_{x}^{0}\left|\mathscr{O}_{n}^{x}\right| B_{x}^{0}\right\rangle=\beta_{n}\left(1+\mathscr{W}_{B}+\mathscr{T}_{x}^{(n)}+\tilde{\mathscr{T}}_{x}^{(n)}\right)+\beta_{n}^{\prime}\left(\mathscr{Q}_{x}^{(n)}+\tilde{\mathscr{Q}}_{x}^{(n)}\right)+\text { analytic terms, }
$$

where $\mathscr{W}_{B}$ is the $B$ wave-function renormalization, $\mathscr{T}$ and $\tilde{T}$ are the right- and wrong-spin tadpole diagrams, and $\mathscr{Q}$ and $\tilde{\mathscr{Q}}$ are the right- and wrong-spin sunset diagrams. In the special case of operator $\mathscr{O}_{1}, \beta_{1}^{\prime}=\beta_{1}$ by a heavy-quark spin argument [13]. 
The diagrams for operator $\mathscr{O}_{1}^{x}$ then give

$$
\begin{aligned}
& \mathscr{T}_{x}^{(1)}=\frac{-i}{f_{\pi}^{2}}\left\{\frac{1}{16} \sum_{\delta, \rho} N_{\rho} \mathscr{I}_{x \delta, \rho}+\frac{1}{16} \sum_{\rho} N_{\rho} \mathscr{I}_{X, \rho}+\frac{2}{3}\left[R_{X_{I}}^{[2,2]}\left(\left\{M_{X_{I}}^{(5)}\right\} ;\left\{\mu_{I}\right\}\right) \frac{\partial \mathscr{I}_{X, I}}{\partial m_{X_{I}}^{2}}\right.\right. \\
& \left.-\sum_{j \in\left\{M_{I}^{(5)}\right\}} D_{j, X_{I}}^{[2,2]}\left(\left\{M_{X_{I}}^{(5)}\right\} ;\left\{\mu_{I}\right\}\right) \mathscr{I}_{j, I}\right]+a^{2} \delta_{V}^{\prime}\left[R_{X_{V}}^{[3,2]}\left(\left\{M_{X_{V}}^{(7)}\right\} ;\left\{\mu_{V}\right\}\right) \frac{\partial \mathscr{I}_{X, V}}{\partial m_{X_{V}}^{2}}\right. \\
& \left.\left.-\sum_{j \in\left\{M_{V}^{(7)}\right\}} D_{j, X_{V}}^{[3,2]}\left(\left\{M_{X_{V}}^{(7)}\right\} ;\left\{\mu_{V}\right\}\right) \mathscr{I}_{j, V}\right]+(V \rightarrow A)\right\} \\
& \tilde{\mathscr{T}}_{x}^{(1)}=\frac{-i}{f_{\pi}^{2}}\left\{\frac{1}{16}\left(-5 \mathscr{I}_{X, P}-4 \mathscr{I}_{X, A}+18 \mathscr{I}_{X, T}-4 \mathscr{I}_{X, V}-5 \mathscr{I}_{X, I}\right)+\frac{2\left(\beta_{2}+\beta_{3}\right)}{\beta_{1}}\left(\mathscr{I}_{X, A}-\mathscr{I}_{X, V}\right.\right. \\
& +a^{2} \delta_{V}^{\prime}\left[R_{X_{V}}^{[3,2]}\left(\left\{M_{X_{V}}^{(7)}\right\} ;\left\{\mu_{V}\right\}\right) \frac{\partial \mathscr{I}_{X, V}}{\partial m_{X_{V}}^{2}} \quad-\sum_{j \in\left\{M_{V}^{(7)}\right\}} D_{j, X_{V}}^{[3,2]}\left(\left\{M_{X_{V}}^{(7)}\right\} ;\left\{\mu_{V}\right\}\right) \mathscr{I}_{j, V}\right] \\
& \left.\left.-a^{2} \delta_{A}^{\prime}\left[R_{X_{A}}^{[3,2]}\left(\left\{M_{X_{A}}^{(7)}\right\} ;\left\{\mu_{A}\right\}\right) \frac{\partial \mathscr{I}_{X, A}}{\partial m_{X_{A}}^{2}}-\sum_{j \in\left\{M_{A}^{(7)}\right\}} D_{j, X_{A}}^{[3,2]}\left(\left\{M_{X_{A}}^{(7)}\right\} ;\left\{\mu_{A}\right\}\right) \mathscr{I}_{j, A}\right]\right)\right\}, \\
& \mathscr{Q}_{x}^{(1)}=\frac{-i g_{B^{*} B \pi}^{2}}{f_{\pi}^{2}}\left\{\frac{1}{16} \sum_{\rho} N_{\rho} \mathscr{H}_{X, \rho}^{\Delta^{*}}+\frac{1}{3}\left[R_{X_{I}}^{[2,2]}\left(\left\{M_{X_{I}}^{(5)}\right\} ;\left\{\mu_{I}\right\}\right) \frac{\partial \mathscr{H}_{X, I}^{\Delta^{*}}}{\partial m_{X_{I}}^{2}}\right.\right. \\
& \left.\left.-\sum_{j \in\left\{M_{I}^{(5)}\right\}} D_{j, X_{I}}^{[2,2]}\left(\left\{M_{X_{I}}^{(5)}\right\} ;\left\{\mu_{I}\right\}\right) \mathscr{H}_{j, I}^{\Delta^{*}}\right]\right\} \\
& \tilde{\mathscr{Q}}_{x}^{(1)}=\frac{-i g_{B^{*} B \pi}^{2}}{f_{\pi}^{2}}\left\{\frac{1}{16}\left(-5 \mathscr{H}_{X, P}^{\Delta^{*}}-4 \mathscr{H}_{X, A}^{\Delta^{*}}+18 \mathscr{H}_{X, T}^{\Delta^{*}}-4 \mathscr{H}_{X, V}^{\Delta^{*}}-5 \mathscr{H}_{X, I}^{\Delta^{*}}\right)+\frac{2\left(\beta_{2}^{\prime}+\beta_{3}^{\prime}\right)}{\beta_{1}}\left(\mathscr{H}_{X, A}^{\Delta^{*}}\right.\right. \\
& -\mathscr{H}_{X, V}^{\Delta^{*}}+a^{2} \delta_{V}^{\prime}\left[R_{X_{V}}^{[3,2]}\left(\left\{M_{X_{V}}^{(7)}\right\} ;\left\{\mu_{V}\right\}\right) \frac{\partial \mathscr{H}_{X, V}^{\Delta^{*}}}{\partial m_{X_{V}}^{2}}-\sum_{j \in\left\{M_{V}^{(7)}\right\}} D_{j, X_{V}}^{[3,2]}\left(\left\{M_{X_{V}}^{(7)}\right\} ;\left\{\mu_{V}\right\}\right) \mathscr{H}_{j, V}^{\Delta^{*}}\right] \\
& \left.\left.-a^{2} \delta_{A}^{\prime}\left[R_{X_{A}}^{[3,2]}\left(\left\{M_{X_{A}}^{(7)}\right\} ;\left\{\mu_{A}\right\}\right) \frac{\partial \mathscr{H}_{X, A}^{\Delta^{*}}}{\partial m_{X_{A}}^{2}}-\sum_{j \in\left\{M_{A}^{(7)}\right\}} D_{j, X_{A}}^{[3,2]}\left(\left\{M_{X_{A}}^{(7)}\right\} ;\left\{\mu_{A}\right\}\right) \mathscr{H}_{j, A}^{\Delta^{*}}\right]\right)\right\} .
\end{aligned}
$$

The chiral logarithm functions $\mathscr{I}$ and $\mathscr{H}^{\Delta^{*}}$ are defined in Ref [13], the subscripts on these functions give the flavor and taste of the relevant meson, $\mathcal{S}$ runs over the sea quarks, $\rho$ sums over taste representations $P, A, T, V, I$ (with degeneracies $N_{\rho}$ ), $X$ is the $x \bar{x}$ meson, and other notation is given in Refs. $[7,12]$. The contributions to operators $\mathscr{O}_{2}-\mathscr{O}_{5}$ have similar forms.

\section{Discussion}

Wrong spin/taste operators arise from the local operator construction and are $\mathscr{O}(1)$ in the lattice spacing. Their contributions to matrix elements are suppressed to NLO because taste-symmetry violation is required. This is different from mixings due to perturbative corrections, which are suppressed by $\alpha_{S} / 4 \pi$. One-loop in $\chi$ PT then makes them $\mathscr{O}\left(a^{2} \alpha_{S} / 4 \pi\right)$, which is effectively NNLO. So non-analytic chiral logarithms do not arise at NLO from the perturbative corrections. A similar 
statement is true for possible $\mathscr{O}(a)$ and higher terms in the relation between the naive quarks and the spin-taste basis.

The wrong-spin effects induce mixing in the $\chi \mathrm{PT}$ of various operators. However, as long as all five $\mathscr{O}_{n}$ are analyzed simultaneously, there are no new low-energy constants induced by these effects: the $\beta_{n}$ and $\beta_{n}^{\prime}$ are all already present in the continuum.

References [3, 4] focused on the calculation of the quantity $\xi \equiv\left(f_{B_{s}} \sqrt{\hat{B}_{B_{s}}}\right) /\left(f_{B_{d}} \sqrt{\hat{B}_{B_{d}}}\right)$, which comes from the matrix element of operator $\mathscr{O}_{1}$. The chiral effects of the wrong spins were not known at the time of the HPQCD calculation [3] and were therefore omitted from the analysis and error estimate. In the Fermilab/MILC calculation [4], the full $\chi \mathrm{PT}$ expression was available, but the matrix elements of operators other than $\mathscr{O}_{1}$ were not calculated, preventing a direct inclusion of the wrong-spin effects. However, it was possible to estimate the error of omitting these effects by using a small subset of new data to investigate the other matrix elements. The result, $\xi=1.268(63)$ included a 3.2\% error from this effect, which was the second largest source of error. In the ongoing second-generation Fermilab/MILC project [14], matrix elements of all five operators $\mathscr{O}_{n}$ are being computed, which means that the full $\chi \mathrm{PT}$ expression can be used in the analysis, and there will be no "wrong-spin error." Of course, a chiral/continuum extrapolation error will remain.

I thank J. Laiho, R.S. Van de Water, and C. Bouchard for discussions and for help with various aspects of the calculation. This work has been partially supported by the Department of Energy, under grant number DE-FG02-91ER40628.

\section{References}

[1] F. Gabbiani, E. Gabrielli, A. Masiero and L. Silvestrini, Nucl. Phys. B 477, 321 (1996) [hep-ph/9604387].

[2] C. M. Bouchard, FERMILAB-THESIS-2011-32.

[3] E. Gamiz et al. [HPQCD Collaboration], Phys. Rev. D 80, 014503 (2009) [arXiv:0902.1815].

[4] A. Bazavov, et al. [Fermilab Lattice and MILC Collaborations], Phys. Rev. D 86 (2012) 034503 [arXiv:1205.7013].

[5] G. P. Lepage and B. A. Thacker, Nucl. Phys. Proc. Suppl. 4, 199 (1988); B. A. Thacker and G. P. Lepage, Phys. Rev. D 43, 196 (1991).

[6] A. X. El-Khadra, A. S. Kronfeld and P. B. Mackenzie, Phys. Rev. D 55, 3933 (1997) [arXiv:hep-lat/9604004].

[7] C. Aubin and C. Bernard, Phys. Rev. D 73, 014515 (2006) [arXiv:hep-lat/0510088].

[8] C. G. Boyd and B. Grinstein, Nucl. Phys. B 442, 205 (1995) [hep-ph/9402340].

[9] A. Bazavov, et al. [Fermilab Lattice and MILC Collaborations], Phys. Rev. D 85, 114506 (2012) [arXiv:1112.3051].

[10] M. Wingate, et al., Phys. Rev. D 67, 054505 (2003) [hep-lat/0211014].

[11] F. Gliozzi, Nucl. Phys. B 204, 419 (1982); A. Duncan, R. Roskies and H. Vaidya, Phys. Lett. B 114, 439 (1982); H. Kluberg-Stern, A. Morel, O. Napoly and B. Petersson, Nucl. Phys. B 220, 447 (1983).

[12] C. Aubin and C. Bernard, Phys. Rev. D 76, 014002 (2007) [arXiv:0704.0795].

[13] W. Detmold and C. J. D. Lin, Phys. Rev. D 76, 014501 (2007) [hep-lat/0612028].

[14] E. Freeland et al. [Fermilab Lattice and MILC Collaborations], POS (LAT2012) 124. 